Los hijos de Sánchez es una novela construida a partir de historias narradas oralmente por quienes hasta entonces no tenían voz, y que aqui traducen la historia oficial de México. Las suyas son reescrituras intralingüisticas e interlingüisticas que ejemplifican lo que Bastin (2006: 121) Ilama "oraliture». Este artículo muestra cómo la peculiar historia que reescribe Lewis a través de sus personajes es un ejemplo de esas traducciones de las que habla la historiografía crítica norteamericana y francesa, historias que traducen la Historia desde abajo, microhistorias. Son, pues, traducciones que superan el tradicional eurocentrismo y universalismo, que ofrecía sólo la visión de los vencedores.

PALABRAS CLAVE: traducción, historia, subalternos, Oscar Lewis.

\title{
Los nadies, traductores de la historia: el ejemplo de Los hijos de Sánchez:
}

\author{
Ma Carmen África Vidal Claramonte \\ Universidad de Salamanca
}

* Este artículo forma parte del proyecto de investigación titulado 'Violencia simbólica y traducción: retos en la representación de identidades fragmentadas en la sociedad global', financiado por el Ministerio de Economía y Competitividad (FFI20I5-665I6-P; MINECO/ FEDER).

\section{The nobodies' translations of history: the case of The Children of Sánchez}

Los hijos de Sánchez is an example of a novel constructed by (hi)stories narrated orally by those who did not previously have a voice, and now translate Mexico's official history. Theirs are intralinguistic and interlinguistic rewritings exemplifying what Bastin (2006: 121) calls "oraliture". This article will show that Lewis' particular way of translating history takes as his starting point the concepts of American and French critical historiography. Lewis' "translators" offer rewritings, histories from below, microhistories. This opens new venues to overcome the traditional Eurocentrism and universalism which offered us only the vision of the conquerors.

KEY WORDS: translation, history, subalterns, Oscar Lewis. 
Sueñan las pulgas con comprarse un perro y sueñan los nadies con salir de pobres, que algún mágico día llueva de pronto la buena suerte, que llueva a cántaros la buena suerte; pero la buena suerte no llueve ayer, ni hoy, ni mañana, ni nunca, ni en lloviznita cae del cielo la buena suerte, por mucho que los nadies la llamen y aunque les pique la mano izquierda, o se levanten con el pie derecho, o empiecen el año cambiando de escoba.

Los nadies: los hijos de nadie, los dueños de nada.

Los nadies: los ningunos, los ninguneados, corriendo la liebre, muriendo la vida, jodidos, rejodidos:

Que no son, aunque sean.

Que no hablan idiomas, sino dialectos.

Que no profesan religiones, sino supersticiones.

Que no hacen arte, sino artesanía.

Que no practican cultura, sino folklore.

Que no son seres humanos, sino recursos humanos.

Que no tienen cara, sino brazos.

Que no tienen nombre, sino número.

Que no figuran en la historia universal, sino en la crónica roja de la prensa local.

Los nadies, que cuestan menos que la bala que los mata

Eduardo Galeano (1989/2009: 59).

We fear those who speak about us who do not speak to us and with us. We know what it is like to be silenced. We know that the forces that silence us because they never want us to speak, differ from the forces that say speak, tell me your story.

bell hooks (1991: 343).

Dice Ricardo Piglia en La ciudad ausente que narrar es hacer vivir a quien tiene miedo de vivir. $\mathrm{Y}$, en La literatura en peligro, Todorov concluye que ama la literatura porque le ayuda a vivir, pero no porque le evite las heridas que inevita- blemente se sufren con el contacto con los otros sino porque le permite descubrir esos mundos de los otros y entenderlos mejor. La literatura no nos anestesia; más bien todo lo contrario. Como afirmó Mario Vargas Llosa (2015) en su Discurso de investidura como Doctor Honoris Causa por la Universidad de Salamanca el i7 de septiembre, la literatura es «una fuente de insatisfacción permanente [que] crea ciudadanos descontentos inconformes» y que en consecuencia «[n]os hace a veces más infelices, pero también muchísimo más libres». En el campo de la ficción, viene enseguida a la memoria Fabrenheit 45I, la novela distópica de Ray Bradbury que ya en I953 describe un país en el que se prohíbe leer porque la lectura obliga a pensar, lo que impide ser ingenuamente feliz. No en vano el título hace referencia a la temperatura en la escala de Fahrenheit a la que el papel de los libros se inflama y arde. La solución que propone Bradbury son los hombres-libro, aquellos que guardan en su memoria un libro para salvarlo de las llamas, y por eso un señor responde por $E l$ Buscón y una señora es Madame Bovary.

Podríamos seguir citando a muchos escritores para quienes, efectivamente, una de las funciones de la literatura es ser testigo de la historia, de lo que acontece en una sociedad; y ser testigo para contarlo. Pero para contarlo desde todas las perspectivas, la de los vencedores y la de los vencidos. Este es, sin duda, el caso del autor objeto de estudio de este artículo, Oscar Lewis, reconocido por contar las historias de los otros, por dar voz a quienes no la tienen.

El propósito de este trabajo de investigación es analizar una de sus obras más polémicas, Los bijos de Sánchez, desde la perspectiva de la historiografía crítica de los años sesenta, que desconstruyó las ideas hasta entonces imperantes sobre qué era un texto histórico, qué características debía tener para serlo, quién tenía la auto- 
ridad para construirlo y de qué fuentes debía beber. Todos esos puntos de partida son los que renuevan los historiógrafos que serán el fundamento de nuestra hipótesis, en tanto entienden el texto histórico como una narración, como una reescritura de la realidad. A partir de ahí, el historiador pasa a ser un traductor de los hechos que acontecieron en el pasado.

\section{LA NUEVA MANERA DE ENTENDER LA HISTORIA}

Tradicionalmente, desde Leopold von Ranke, "proper" history is seen to be that which is accepted as such by "proper" historians" (Morley, 1999/2002: 22). Para serlo, un texto histórico debía estar construido a partir de determinados archivos y fuentes oficiales y escrito por alguien con la suficiente autoridad y capital simbólico reconocidos. Por eso se consideraba que la historia era una disciplina objetiva que contaba lo acontecido en el pasado de una manera neutral.

Frente a esta visión decimonónica de la historia, son muchos los historiadores que desde corrientes y metodologías diferentes sospechan de la historiografía tradicional y entienden la historia como un texto, como una narración, la narración de alguien, que por lo tanto nunca es neutral. Por decirlo con Alun Munslow (2013: 6), "the story" in history is provided by the historian».

Desde mediados de los años sesenta del pasado siglo, ha habido diferentes escuelas desde la historiografía crítica norteamericana, los Estudios Subalternos de la India hasta la Escuela de los Anales francesa o la italiana de las microhistorias - que han supuesto una verdadera revolución en la manera de entender y definir qué es un texto histórico y sobre todo quién puede escribirlo o contarlo. Son metodologías diferentes entre sí pero que comparten la idea de que la historia no es neutral y objetiva como deseaban los historiadores decimonónicos con van Ranke a la cabeza, sino que se debe escribir la historia de todos (para una análisis mucho más detallado de todas estas nuevas maneras de construir la historia, véase Rundle, 20I8, 20I4, 20I2; Rundle y Rafael, 2016; Vidal, 2018).

Los historiadores se van dando cuenta de que la pretensión de objetividad y neutralidad de la disciplina no es sino una imposición de quienes están en el poder, que esperan que se acepte su construcción de la historia como la única narración verdadera. La historia se ha contado hasta hace relativamente poco desde lo que Hayden White (I987: 20) llama la «doxa» del «establisbment historiográfico», una perspectiva que intentaba borrar las huellas del sujeto, sus circunstancias particulares, su ideología en suma, y que entendía por tanto que el discurso carecía de sesgo alguno y que el relato era equivalente a los hechos.

Lo que las nuevas voces desean es poner en un brete las definiciones anteriores y dejar claro que «there is no such thing as a neutral, unproblematic definition of history; all definitions are working to someone's advantage at someone else's expense. To the question "What is history?", we must always add: "Who decides? On what grounds, and to what end?"» (Morley, 1999/2002: 23). La historia se convierte así en una forma de representación de la realidad (LaCapra, 20I3), en una construcción donde las verdades son "useful fictions" that are in discourse by virtue of power (somebody has to put and keep them there) and power uses the term "truth" to exercise control: regimes of truth" (Jenkins, I99I/2003: 39).

Gracias a que la historiografía contemporánea se ha abierto a nuevas formas de representación y a nuevas metodologías y escuelas como

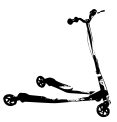


las mencionadas, cada vez se hace más evidente que historias hay muchas, que la historias son historias, textos que dependen de las palabras (Hayden White, 1987) y de las fuentes que elige el historiador para describir el pasado. Lejos de ser una actividad objetiva, es selectiva. Y esa selección se hace con palabras: «somos las palabras que cuentan lo que somos» (Galeano, 1989/2009: 4). La historia es un artefacto literario (Hayden White, I978a, 1978b), y como tal el traductor deberá tener muy en cuenta las muchas maneras en las que la producción de narrativas históricas revela la asimetría entre grupos e individuos que tienen acceso muy diferente a los medios de producción de la historia (Trouillot, I995: XIX).

Es en ese momento cuando según Hayden White el historiador se convierte en traductor de la realidad; porque es quien hace una primera traducción intralingüística. Se trata de una manera de abordar el texto histórico tan novedosa como controvertida: si la historia es una narración, es también una reescritura de los hechos, una traducción del pasado que narra un historiador/traductor concreto en función de las palabras y de las fuentes que elige. En Metahistory (I975: I29) Hayden White afirma explícitamente que el texto histórico es una manera entre otras de traducir la realidad, y en consecuencia que el historiador es un traductor. En The Content of Form (1987) se define la historia como «the problem of how to translate knowing into telling» (White, I987: I). Y otro importante historiador, Reinhart Koselleck (2002: VIII), considera que la historia de la historiografía es una historia de la evolución del lenguaje de los historiadores. La historia es un «act of translation» (Jenkins 199I/2003: 48). Es más, en este contexto, Hayden White asegura que la historia se convierte en una manera muy eficaz de desarrollar la autorreflexión (D'hulst y Gambier, 2018: 3), algo que Gambier (2007) aplicó hace ya tiempo al campo de la traducción.

Será desde esta manera de entender el texto histórico como nos aproximaremos a The Children of Sánchez primero y a su traducción al español después.

\section{TRADUCIR LA HISTORIA DE MÉXICO DESDE ABAJO: THE CHILDREN OF SÁNCHEZ}

Oscar Lewis es un autor preocupado desde siempre por quienes no tienen voz. A mediados de la década de los cincuenta, este interés por la pobreza de los barrios de la ciudad de México se publicó en forma de su Antropología de la pobreza. Cinco familias (1959 en inglés, 196r en español) y Los hijos de Sánchez (I96I en inglés, I964 en español), dos obras en las que Lewis combinó la investigación antropológica con un particular estilo escritural al que llamó «realismo etnográfico».

The Children of Sánchez es un clásico de la antropología moderna. Es uno de los ejemplos más claros de historia contada desde abajo, desde la voz de quienes hasta entonces eran «nadies», por decirlo con Galeano (1989/2009: 59). Es un conjunto de microhistorias, una narración de las otras historias, de las historias de los otros, contadas desde puntos de vista que no son los oficiales. Es, según las ideas de la historiografía crítica antes mencionadas, una traducción intralingüística de la historia oficial.

La obra de Lewis es también una crónica, ese género latinoamericano que habla de «a marginal reality, bringing to the fore aspects of city life that have tended to be ignored and articulating discourses from disempowered social groups» (Bielsa, 2006: xIv). Asimismo se puede entender como novela testimonio, narrativa documental, novela de no ficción, crónica documental, género testimonio, novela 
sin ficción: «These terms are not used in an entirely interchangeable fashion, although a single text may be classified in a number of different ways. Testimonio and testimonial literature are the two most widely used terms to designate a whole group of structurally and thematically diverse texts» (Jörgensen, 1994: I47). Una magnífica definición de testimonio la da Gugelberger (1996: 9), quien afirma que el testimonio «becomes interesting not so much for what it says and how it says it (as literature per se), but rather for how it entered critical discourse and the institutional centers of higher learning, thereby dismantling our treasured notions of literature».

Uno de los rasgos más llamativos de estas reescrituras de la historia desde abajo, llamémoslas testimonios o crónicas, es que se basan en la oralidad (Thompson, I966; Thompson, I978). Recordemos que la tradición oral era para Walter Benjamin (I936/I99I: III-I34) la clave de toda narración. La oralidad es subversiva porque es múltiple y plural, porque se ha relacionado con quienes se consideran inferiores intelectualmente, quienes en consecuencia no han podido poner por escrito sus historias. $\mathrm{La}$ oralidad es hoy en día objeto de estudio en muchas disciplinas, entre ellas la traducción, que se ha empezado a dar cuenta de la importancia de traducir éticamente esas historias orales, que en ocasiones pueden ser muy conflictivas (Bandia, 2015; McDonough Dolmaya, 2018 y 2015; Thompson, 1978; Reeves-Ellington, I999; Cifuentes-Goodbody y Harding, 20I6).

En este caso, Oscar Lewis da la palabra a los miembros de la familia Sánchez (que en realidad era la familia Hernández, porque su identidad se debía mantener oculta): el patriarca viudo, Jesús Sánchez, y sus cuatro hijos (Manuel, Roberto, Consuelo y Marta, cuyos nombres son ficticios), habitantes de Tepito, donde cada cuarto de vecindad en la década de los cincuenta se caracterizaba por alojar a familias muy extensas, con varias generaciones, a pesar de que eran viviendas de una sola estancia, con baño compartido con otras muchas familias. En la edición inglesa de la obra, Lewis dice que la vecindad (una palabra que siempre aparece en español y en cursiva) se llama La Casa Grande, mientras que en la traducción se denomina Bella Vista. Ninguno de los dos son el verdadero nombre, que era La Casa Blanca. Las voces hasta ahora silenciadas reescriben la realidad de México que la historia oficial no quiere contar a través de sus vivencias, desde su niñez hasta la edad adulta, y como resultado traducen esa realidad y esa historia oficial de una manera, según algunos (aunque afirmar esto es evidentemente conflictivo), más ética.

En The Children of Sánchez encontramos microhistorias, narraciones en el sentido antes mencionado de Hayden White, que cuentan el pasado mexicano mientras los personajes relatan la historia de sus vidas a lo largo de cinco monólogos. Como señala el propio autor en su introducción al libro,

I offer the reader a deeper look into the lives of one of these families by the use of a new technique whereby each member of the family tells his own life story in his own words. This approach gives us a cumulative, multifaceted, panoramic view of each individual, of the family as a whole, and of many aspects of lower-class Mexican life (Lewis, 196r/20II: XXIII).

Es interesante fijarse en que Lewis subraya que cada miembro de la familia elige unas palabras diferentes para contar la realidad desde su punto de vista. Es decir, en la línea de Hayden White, Koselleck o Jenkins, nos avisa de que cada uno es un autor, pero también un traductor de los hechos acontecidos en sus vidas y en sus con-

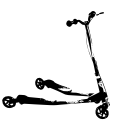

101 
textos. Sería muy interesante para los estudios de traducción y concretamente para las nuevas maneras de hacer historia de la traducción analizar en profundidad esos cambios terminológicos, esas palabras diferentes que elige cada narrador, cada autor, cada traductor de la realidad, y al estudiarlo pensar por qué, qué influye para que, por ejemplo, las palabras de las hijas sean diferentes a las de sus hermanos. Son estas microhistorias, todas juntas, las que nos hacen percibir una idea más veraz de lo que estaba ocurriendo en México en ese momento:

The independent versions of the same incidents given by the various family members provide a built-in check upon the reliability and validity of much of the data and thereby partially offset the subjectivity inherent in a single autobiography. At the same time it reveals the discrepancies in the way events are recalled by each member of the family (Lewis, I96I/20II: XXIII).

La traducción que cada uno de estos microhistoriadores hace de la historia oficial incluye episodios de una existencia urbana brutal, de un México cuya vida cotidiana es, para muchos, atroz y violenta.

The Children of Sánchez tuvo un proceso de escritura muy interesante. Lewis grabó a sus protagonistas, lógicamente en el español de México, pero la historia salió publicada originalmente en inglés en i96r. En su introducción a esa versión inglesa el autor habla del proceso de traducción llevado a cabo del español al inglés. Lewis reconoce la importancia y la responsabilidad ética de traducir bien las historias orales —que, como se ha señalado antes, en español son, según la historiografía crítica, ya de por sí, una traducción de la historia oficialpero también lo difícil que fue para él traducirla, por el tipo de lenguaje que muchas veces se utiliza en estos relatos. Lewis reconoce los fallos de la traducción interlingüística:

The translation of lower class Mexican Spanish has presented formidable and in some ways insoluble problems, particularly in attempting to find equivalents for slang expressions, idioms, and jokes with sexual innuendo. I have tried to capture the essential meaning and flavor of the language rather than to render a literal translation. Inevitably, some of the unique quality and charm of the original as well as the personal style of each individual has been lost. The English translation gives a surprisingly high level of language and vocabulary to relatively unlettered people. The fluency of language and the vocabulary of Mexicans, be they peasants or slum dwellers, have always impressed me (Lewis, I96I/20II: XXXIV).

Lewis procede aparentemente como un autor que se limita a trasponer en papel lo que le dicen sus entrevistados. En la introducción a la versión inglesa, aseguraba:

This method of multiple autobiographies also tends to reduce the element of investigator bias because the accounts are not put through the sieve of a middle-class North American mind but are given in the words of the subjects themselves (Lewis, I96I/20II: XXIII).

Sin embargo, esa aparente neutralidad no es tal, porque seleccionó la información obtenida con la grabadora, con el trabajo de campo etnográfico, la aplicación de tests psicológicos, la elaboración de cuestionarios, la consulta de archivos, la utilización de fotografías, entre él y sus ayudantes. Aunque Lewis se acerca con respeto al español mexicano de los hablantes y a todas sus variantes y registros, es él (en función de su experiencia, valores y personalidad) quien elige y ordena la información de quienes contaban sus historias: 
In the course of the interviews I asked hundreds of questions of Manuel, Roberto, Consuelo, Marta, and Jesús Sánchez. Naturally, my training as an anthropologist, my years of familiarity with Mexican culture, my own values, and my personality influenced the final outcome of this study [...] In preparing the interviews for publication, I have eliminated my questions and have selected, arranged, and organized the materials into coherent life stories $[\ldots]$ The editing has been more extensive in some cases than in others. Manuel, by far the most fluent and dramatic storyteller in the family, needed relatively little editing [...] The Manuel story perhaps more than the others, however, loses a great deal in transcription and translation because he is born actor with a great gift for nuance, timing, and intonation (Lewis, I96I/20II: XXXIV).

En este caso, Lewis reconoce que el significado no solo surge de las palabras que se eligen sino de cómo se cuentan las historias, porque todo significa, la proxémica, lo no verbal, la música, el ritmo, los silencios. Eliminar o alterar de algún modo esos signos de la oralidad implica irremediablemente manipular el significado de esa microhistoria.

En este sentido, es interesante mencionar que uno de esos ayudantes con los que contó Lewis para sus obras fue Elena Poniatowska, que colaboró en la transcripción de las entrevistas en las que se basaría Pedro Martinez y que reconoce la influencia de Lewis en su forma de escribir y reescribir la historia sobre todo en su conocida novela, Hasta no verte Jesús mío (I969), en la que utilizó por primera vez el procedimiento periodístico de la entrevista, grabando a sus informantes, igual que el antropólogo. Lo que quisiera resaltar aquí es lo que Poniatowska tuvo que hacer para Lewis a propósito de Pedro Martínez, que no fue ni más ni menos que reescribir, manipular, las historias orales de quienes las narraban.
Dicho por ella misma, «limpiar esos relatos de su hojarasca; es decir, eliminar las repeticiones y divagaciones inútiles. Esta experiencia sin duda ha de haberme marcado al escribir Hasta no verte Jesús mio» (Poniatowska, I978: 8). ${ }^{\mathrm{I}}$

La publicación de la versión española de Los hijos de Sánchez por el Fondo de Cultura Económica (FCE) en I964 se hizo a partir de las grabaciones originales en el magnetofón de los entrevistados y de las versiones taquigráficas que hizo el autor. Estamos, pues, ante un proceso tan interesante como conflictivo de reescritura de reescrituras: primero el texto se publica como «original» en inglés, y es en realidad una versión manipulada que pierde muchas de las connotaciones del lenguaje original, según ha reconocido el propio Lewis. Y posteriormente se publica el «original», que es en realidad la traducción intralingüística que hacen los subalternos de la historia de México, según entiende la historiografía crítica de los años sesenta la construcción de la historia. Se trata, por tanto, de una obra que va cobrando forma a base de varios niveles de traducción, intratextual, intertextual, lo que la convierte en una verdadera subversión de las oposiciones binarias tradicionales (original/traducción, autor/traductor, primario/secundario, etc.), dado que nos obliga como lectores a preguntarnos: ¿quién es real-

I Lewis no influyó solamente en su manera de escribir, sino también en el contenido de sus novelas (Poniatowska, 1978; Gardner, 2007: 34-37 y 62-67). Las dos obras importantes que había publicado eran un libro de historias cortas sobre una chica mexicana burguesa, Lilus Kikus (1954), y Palabras cruzadas (I96I), un libro de entrevistas con importantes intelectuales y artistas mexicanos. Fue Lewis quien le hizo ver la importancia de hablar de los más desfavorecidos y las consecuencias de reescribir y traducir la historia de los vencedores a partir de las palabras de los vencidos. Existen, no obstante, diferencias entre ellos (Gardner, 2007: 63ss): por ejemplo, las relaciones con sus entrevistados y, tal vez más importante en el caso de este estudio, que las obras de Poniatowska tienen más forma de novela que de documento antropológico o social.

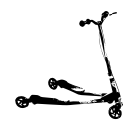

103 
mente el autor o autores de The Children of Sánchez? ¿Es el mismo/los mismos que Los hijos de Sánchez? ¿Quién la ha traducido? Como señala muy acertadamente Juan Jesús Zaro (2014), en ningún sitio se menciona si la versión de la obra en español consistió en transcribir las cintas o si hubo algo más. Entonces, ¿cuál es en realidad el original? ¿Qué historia cuentan? ¿Hay una sola historia? ¿Cuál de ellas es la «verdadera»? ¿Existe lo real, la historia real, o sólo realidades, las que cada uno de nosotros construye con el lenguaje?

También resulta muy interesante recordar que la publicación en español de la novela causó, como es bien sabido, un extraordinario revuelo en México.

[e]l gobierno mexicano presidido por Gustavo Díaz Ordaz, escandalizado ante un retrato tan crudo y veraz de la sociedad mexicana, destituyó a Arnaldo Orfila, el editor argentino que desde 1948 había sido director general del Fondo de Cultura Económica, y responsable de la publicación de la obra. Orfila, apoyado por lo más granado de la intelectualidad mexicana, avergonzada por la decisión gubernamental, pasó a dirigir una nueva editorial, Siglo xxi, fundada en ig66. Lo interesante de este episodio, prescindiendo del trauma que provocó en la cultura mexicana de los sesenta, es el extraordinario poder simbólico que adquirió desde entonces la obra, cuya publicación marca un antes y un después en la crónica interna del Fondo de Cultura Económica, pero también en la historia del libro en México (Zaro, 20I4: n.p.).

El libro de Lewis ha interesado en muchos campos además del de la historia, desde la filosofía hasta la sociología, la literatura o la antropología. Michel De Certeau (I984: I59), por ejemplo, se refiere a Los hijos de Sánchez como una obra pionera «que da el habla» y es punto de partida de tantas «historias de vida». Las historias de la obra de Lewis son, según este autor (De Certeau 1984, I986/2010), «heterologías», ciencias del otro, porque intentan escribir la voz. Es una reivindicación de la oralidad. Sin embargo, esta novela no es la única. Con anterioridad, Lewis había publicado estudios sobre lo que él llamó «the subculture of poverty», que reflejan las historias de las clases más bajas de la ciudad y el campo mexicanos. Five Families: Anthropology of Poverty, publicada en inglés en I959 y traducida en I96I, y posteriormente, la ya mencionada Pedro Martinez: A Mexican Peasant and His Family, de i964, son estudios hechos a partir de la oralidad que querían también reescribir y traducir la historia oficial de México. En esta misma línea, en I966 ve la luz La Vida: A Puerto Rican Family in the Culture of Poverty, uno de los libros más dramáticos y reveladores que se hayan escrito sobre la pobreza y la miseria de los desheredados en un país latinoamericano. Es un libro que se ha clasificado, desde la antropología, dentro de los llamados «estudios de familia». La familia Ríos es una microhistoria que refleja toda una cultura de la pobreza. La Vida es, una vez más, la reescritura de la Historia oficial a través de las historias orales contadas por sus protagonistas:

The tape recordings of the life histories were begun only after we knew the family well. In some cases we visited the family regularly for a few months and learned a great deal about their lives in casual conversations. Later, in the recordings, we would ask the informants to repeat stories which we already knew so that we could have them in their own words (Lewis, I967: xx).

Las narraciones orales de quienes hasta entonces habían sido silenciados son en estas novelas reescrituras (Lefevere, I992) contra el 
poder, traducciones intralingüísticas de una realidad que la cultura dominante, en ejercicios cargados de violencia simbólica, había querido imponer como la única verdadera. Estas historias de naturaleza intrínsecamente conflictiva traducen los hechos desde la perspectiva del subalterno, recuperando así historias olvidadas de quienes no tenían voz pero que no se han resignado a perderla. Son historias que también podrían clasificarse dentro de los llamados «Subaltern Studies», por ser un caso claro de "politically motivated historiography» (Chakrabarty, 20I6: 6r).

\section{EL CONFLICTO DE TRADUCIR UN TEXTO HISTÓRICO}

El giro radical que desde hace décadas ha dado la historiografía, tanto en su manera de definir la historia como de entender cómo se construye un texto histórico, quién lo hace, con qué medios, desde qué ideología, como ha quedado brevemente descrito en la primera sección de este artículo, ya está hoy plenamente aceptado en la disciplina de la historia. Sin embargo, estos cambios nos interesan especialmente porque también los estudios de traducción han estado muy atentos a las innovaciones en una disciplina que nos queda muy cercana, en tanto el discurso historiográfico se construye con actos lingüísticos que traducen la realidad, y que, como toda traducción, ocurren en un contexto determinado y no en el vacío. Martha Cheung, por ejemplo, subraya que sendas disciplinas comparten la misma epistemología y la misma crisis de las Grandes Narrativas: «just as the reliability of historical "facts" is assumed, so too is the reliability of translation as a "faithful" reflection of a factual "original". Yet that epistemology... is very much in crisis» (Cheung, 20I2: 158$)$ :
If knowledge is mediated, this is all the more true of historical knowledge... But most heavily mediated is knowledge gained in the domain of translation history... historical studies on translation depict sites of vigorous contestations. They show how overdetermined works of translation often are - by the producers of grand narratives of national identity as much as by marginalized groups, hybrid groups, and by invaders, explorers, travelers, colonial administrators, missionaries, linguists, anthropologists, spies, and other such information-gartherers (Cheung, 2012: 156, 157).

Y un historiador como Vicente Rafael, en cuyos libros tan importante es la política de la traducción y del lenguaje en la construcción de la historia, asegura que la traducción es crucial «in the workings of colonial history» y en general «in the formation of historical imagination». La traducción, afirma, ayuda a contar «a different history»:

From the perspective of histories of the relationship between self and society, or society and the State, translation is this generalized process differentiation - from within as well as across languages and societies, the study of which allows us to get beyond historicist accounts of institutions and "big men"» (Rafael, en entrevista con Christopher Rundle, en Rundle y Rafael, 2or6: 26).

En el mismo artículo, Rafael no duda en afirmar, desde su condición de historiador, que prefiere «an approach from below, working with the materiality of texts and the landscape of documental evidence to show how historical investigation will always be linked to the workings of translation in one form or another» (ib.: 29). De hecho, advierte que el que las culturas colonizadas hayan mantenido ciertas palabras sin traducir, sin someterse a la lengua

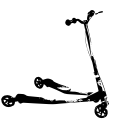

105 
del colonizador (lo que él llama «the insurgency of language») es señal de que la conquista nunca ha llegado a ser completa:

There is always resistance. There is the permanent possibility of a war of meaning against those who seek to use translation to prop up structures of power... Translation does not convey meaning whole and untouched; rather it inflects and distorts it, leaving it open-ended, hence, available for on-going revision, subversion and supplementation... There is in the very working of untranslatability the hope for some other possibility to emerge apart from dominant structures of power. But there is also the risk that it may not, and what happens is that a more sophisticated, more penetrating and more comprehensive set of power relations emerge (ib.: 33 ).

Los historiadores de la traducción están empezando a asumir, pues, todos estos cambios ya asentados en la historiografía, y a entender el profundo conflicto al que se enfrenta el traductor en textos como el que se ha escogido en este artículo, porque son textos que desconstruyen las oposiciones binarias entre autor/traductor, primario/secundario, y alertan del riesgo de que cualquier posibilidad de aproximación a la historia contada desde abajo termine siendo esencialista, eurocéntrica, colonialista o asimétrica. Ante estos escritos, nuestra forma de traducir nos delata. Se trata de hacerle preguntas nuevas al texto de las que ya nos advirtieran tras el giro cultural:

«What is the function of the (this, not a, any) translation likely to be?» "What type of text needs to be translated?» «Who is the initiator of the/this translation?»... The questions that now dominate the field are able to dominate it because research has taken a «cultural turn», because people in the field began to realise, some time ago, that translations are never produced in a vacuum, and that they are also never received in a vacuum (Bassnett y Lefevere, I998: 3).

Efectivamente, como afirma el magnífico monográfico de Meta (2005) editado por Georges Bastin ya hace más de una década, traducir al otro es re-presentarnos. En esa misma línea, autores como Paul Bandia y Georges Bastin o Charles Nama han iniciado una interesantísima y muy novedosa línea de investigación en la cual, a la hora de hacer historias de la traducción, incorporan temas como la otredad, la ideología, el género, la asimetría, la manipulación, la ética, el poscolonialismo o las minorías (Bandia en Bastin y Bandia, 2006: 54) y visiones anti-rankeanas (Bandia, 2009), historias de la traducción desde abajo (Munday, 20I4), basadas en la genealogía foucaultiana (Belle 20r4) y no eurocéntricas (Bastin, 20I7), empeñados como están en estudiar y sistematizar historias de la traducción hasta ahora olvidadas (por ejemplo, Nama, I990 y I993; o Cheung, 2009).

Lógicamente, abordar así la historia torna la cuestión de la traducción de textos históricos y de la construcción de nuevas maneras de ver la historia de la traducción en algo tan apasionante como conflictivo:

The debate between the modernist empiricists and the postmodernist deconstructionists can constitute a basis for discussing some fundamental questions of methodology in translation history: what is the role of the translation historian in documenting or recreating the past? Is translation history, as a discipline, a mere recounting of past events, a deciphering of the traces of the past, so to speak? Or should the discipline be construed as serious historiography, with a decidedly interventionist role for the translation historian? (en Bastin y Bandia, 2006: 48). 
Bandia, por ejemplo, llama al activismo:

The point here is to call on translation historians to engage in an activist paradigm shift.. and to avoid the practice of apolitical historicism or purported objectivity or neutrality in historical narration (Bandia, 20I4: II3).

Efectivamente, para traducir con responsabilidad textos como los de Lewis mencionados aquí pero también otros muchos, habrá que hacerse primero muchas preguntas:

Whose stories are told, from whose perspective, in whose voice? Whose stories, versions, and voices are left silent? Translated? Warped?... Will history be rewritten? What structure will its plot assume? Whose tale will be told above and over all others as the official story? Whose voices will whisper around the edges of the canon, telling their heretical versions? Whose voices will be forcibly silenced, and whose will die out? (Price, 2004: XIII y XVIII).

Los avances en historiografía y en los estudios de traducción demuestran que estamos en el buen camino de empezar a traducir los textos históricos asumiendo antes todas esas preguntas, con el fin de poder así plantear esa manera que parece ser la adecuada para llegar a traducir éticamente: traducir pasando el cepillo de la historia a contrapelo, como dice Walter Benjamin (1955/2005) en su tesis VII, que no consiste en otra cosa que reivindicar, tanto por parte del traductor como del historiador,

...la multiplicidad y densidad constructiva del pasado, constituido siempre por diferentes líneas, proyectos, perspectivas y propuestas en eterno conflicto, líneas y proyectos que encarnando diferentes desarrollos posibles, y diversas evoluciones concretas de la sociedad y de la historia, se enfrentan constantemente en batallas sociales, políticas, culturales, económicas, artísticas, históricas, etc., para ir dibujando y decidiendo, desde los resultados de este conflicto permanente, el verdadero curso de la historia... si la historia no se reduce a la historia del grupo de los vencedores, y si el pasado no es sólo la reivindicación de la tradición y de los hechos orquestada por estos dominadores que han ganado, entonces la tarea del historiador crítico consiste también en reivindicar y rescatar a todos esos pasados vencidos que, a pesar de haber sido derrotados, continúan vivos y actuantes, determinando una parte muy importante de la historia, subterránea y reprimida pero presente dentro del devenir histórico (Aguirre Rojas, 2006: 39-40).

Los microhistoriadores de Lewis son los hombres-libro que se niegan a que el Poder destruya sus historias: por eso las cuentan, por eso traducen su realidad, evitando así el peligro de una sola historia, del que ya advirtiera Chimamanda Ngozi Adichie en una conferencia de 2009 titulada The Danger of a Single Story, donde afirma que la historia única crea estereotipos: «They make one story become the only story», y la historia única «robs people of dignity». Que haya una sola historia, que nos intenten convencer de que sólo hay una historia, nunca es casual:

It is impossible to talk about the single story without talking about power. There is a word, an Igbo word, that I think about whenever I think about the power structures of the world, and it is «nkali». It's a noun that loosely translates to «to be greater than another». Like our economic and political worlds, stories too are defined by the principle of nkali: How they are told, who tells them, when they're told, how many stories are told, are really dependent on power. Power is the ability not just to tell the story of another person, but to make it the definitive story of that person (Adichie, 2009).

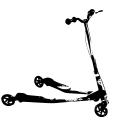

107 
La traducción de lo real da lugar a muchas historias que reflejan muchos puntos de vista y muchas realidades. La traducción interlingüística de dichas realidades traerá consigo una responsabilidad ética acaso mayor, porque añadirá otra microhistoria, la del propio traductor. Lo importante es ser conscientes de que el lenguaje nos delata, y de que, a pesar de nuestras limitaciones, debemos ponernos en lugar del otro con todos sus matices, silencios, ruidos y colores, para intentar en la medida de lo posible construir muchas historias, todas las posibles, porque la diferencia es siempre enriquecimiento:

When we reject the single story, when we realize that there is never a single story about any place, we regain a kind of paradise... Stories matter, many stories matter. Stories have been used to dispossess and to malign, but stories can also be used to empower and to humanize. Stories can break the dignity of a people, but stories can also be used to repair that broken dignity (Adichie, 2009).

RECIBIDO EN SEPTIEMBRE DE 2018 ACEPTADO EN MAYO DE 2019 VERSIÓN FINAL DE JUNIO DE 2019

\section{REFERENCIAS BILIOGRÁFICAS}

Adichie, Ch. N. (2009): «The Danger of a Single Story», TED Talks, julio, <www.ted.com/talks/ chimamanda_adichie_the_danger_of_a_single_story> [consulta: $2-\bar{V}-2018]$.

Aguirre Rojas, Carlos Antonio (2006): Retratos para la historia. Ensayos de contrahistoria intelectual, México: Contrahistorias.

Bandia, Paul (2006): «African Europhone Literature and Writing as Translation. Some Ethical Issues», en Theo Hermans (ed.), Translating Others. Volume 2, Manchester: St. Jerome, 349-364. BAndia, Paul F. (2009): «Cheikh Anta Diop: Translation at the Service of History», en Paul F. Ban- dia y John Milton (eds.), Agents of Translation, Ámsterdam/Filadelfia: John Benjamins, 209-227.

Bandia, Paul F. (2014): «Response», The Translator, 2O/I, II2-II8.

Bandia, Paul F. (20I5): «Introduction: Orality and Translation», Translation Studies, 8 (2), I25-I27.

Bassnett, Susan, y André Lefevere (I998): Constructing Cultures: Essays on Literary Translation, Clevedon: Multilingual Matters.

Bastin, Georges L. (2006): «Subjectivity and Rigour in Translation History: The Latin American Case», en George Bastin y Paul Bandia (eds.), Charting the Future of Translation History, Ottawa: University of Ottawa Press, III-I29.

Bastin, Georges L. (2017): «Eurocentrism and Latin Americanism in Latin American Translation History», Perspectives, 25/2, 260-272.

Bastin, Georges L., y Paul Bandia (eds.) (2006): Charting the Future of Translation History, Ottawa: University of Ottawa Press.

Belle, Marie-Alice (20I4): «At the Interface between Translation History and Literary Translation: A Genealogy of the Theme of "Progress" in Seventeenth-Century English Translation History and Criticism», The Translator 20/1, 44-63.

Benjamin, Walter (1955/2005): Tesis sobre la filosofía de la bistoria y otros fragmentos, trad. Bolívar Echeverría, México: Contrahistorias.

Benjamin, Walter (1936/I99I): «El narrador», trad. Roberto J. Blatt, Para una crítica de la violencia y otros ensayos. Iluminaciones IV, Madrid: Taurus.

Bielsa, Esperança (2006): The Latin American Urban Crónica. Between Literature and Mass Culture, Oxford: Lexington.

Chakrabarty, Dipesh (2016): «Place and Displaced Categories, or How We Translate Ourselves into Global Histories of the Modern», en Doris Backmann-Medick (ed.), The Trans/National Study of Culture. A Translational Perspective, Berlin/ Boston: De Gruyter, 53-68.

Cheung, Martha (20I2): «The Mediated Nature of Knowledge and the Pushing-Hands Approach to Research on Translation History», Translation Studies, 5/2, 156-17I.

Cifuentes-Goodbody, Nicholas y Sue-Ann HarDING (20I6): «An Integrated Approach to the Translation Studies Curriculum», Current Trends in Translation Teaching and Learning, 3, 1-23. 
De Certeau, Michel (1984): The Practice of Everyday Life, trad. Steven Randall, Berkeley: Berkeley University Press.

De Certeau, Michel (1986/2010): Heterologies. Discourse on the Other, trad. Brian Massumi, Minneapolis: University of Minnesota Press.

D'hulst, Lieven e Yves Gambier (eds.) (20i8): A History of Modern Translation Knowledge, Ámsterdam: John Benjamins.

Galeano, Eduardo (1989/2009): El libro de los abrazos, Madrid: Siglo XXI.

Gambier, Yves (2007): «Y a-t-il place pour une socio-traductologie?», en Michaela Wolf y Alexandra Fukari (eds.), Constructing a Sociology of Translation, Ámsterdam: John Benjamins, 205-2I7.

Gardner, Nathanial (2007): Through Their Eyes. Marginality in the Works of Elena Poniatowska, Silvia Molina and Rosa Nissán. Frankfurt: Peter Lang.

Gugelberger, Georg (I996): «Introduction: Institutionalization of Transgression. Testimonial Discourse and Beyond», en Georg Gugelberger (ed.), The Real Thing: Testimonial Discourse and Latin American, Durham: Duke University Press, I-23.

HOOKS, Bell (I99I): "Talking Back», en Russell Ferguson et al. (eds.), Out There: Marginalization and Contemporary Cultures, Nueva York: The New Museum of Contemporary Art; Cambridge, Mass., The MIT Press, 337-344.

Jenkins, Keith (1991/2003): Re-thinking History, Nueva York: Routledge.

Jörgensen, Beth E. (1994): The Writing of Elena Poniatorwsk, Austin: University of Texas Press.

Koselleck, Reinhart (2002): The Practice of Conceptual History: Timing History, Spacing Concepts, Stanford: Stanford University Press.

LaCAPra, Dominick (2013): History, Literature, Critical Theory, Ithaca, NY: Cornell University Press.

Lefevere, André (1992): Translation, Rewriting and the Manipulation of Literary Fame, New York and London: Routledge.

Lewis, Oscar (1961/20II): The Children of Sánchez. Autobiography of a Mexican Family, Nueva York: Vintage.

Lewis, Oscar (1959/2004): Five Families. Anthropology of Poverty, Nueva York: Basic Books.
Lewis, Oscar (1961/2004): Antropología de la pobreza. Cinco familias, México: Fondo de Cultura Económica.

Lewis, Oscar (1964): Pedro Martinez: A Mexican Peasant and His Family, Nueva York: Random House.

Lewis, Oscar (1966): La Vida: A Puerto Rican Family in the Culture of Poverty, Nueva York: Random House.

McDonough Dolmaya, Julie (2015). «A Place for Oral History within Translation Studies», Target, 27/2, I92-2I4.

Morley, Neville (1999/2002): Writing Ancient History, Londres: Duckworth.

Munday, Jeremy (2014): "Using Primary Sources to Produce a Microhistory of Translation and Translators: Theoretical and Methodological Concerns», The Translator, 20, r: 64-80.

Munslow, Alun (ed.) (2013): Authoring the Past. Writing and Rethinking History, Londres/ Nueva York: Routledge.

Nama, Charles Atanganna (1990): "A History of Translation and Interpretation in Cameroon from Precolonial Times to Present», Meta, 35/2, 356-369.

Nama, Charles Atanganna (I993): «Historical, Theoretical and Terminological Perspectives of Translation in Africa», Meta, 38/3, 4I4-425.

Poniatows Ka, Elena (1978): Hasta no verte Jesús mío, Vuelta, 24, 5-II.

Price, Patricia L. (2004): Dry Place. Landscapes of Belonging and Exclusion, Minneapolis/Londres: University of Minnesota Press.

Reeves-Ellington, Barbara (I999): «Responsibility with Loyalty: Oral History Texts in Translation», Target, II/I, IO3-I29.

Rundle, Christopher (20I2): "Translation as an Approach to History», Translation Studies, 5/2, 232-240.

Rundle, Christopher (2014): "Theories and Methodologies of Translation History: The Value of an Interdisciplinary Approach», The Translator, 2O/I, 2-8.

Rundle, Christopher (2018): «Temporality», en Lieven D'hulst e Yves Gambier (eds.), A History of Modern Translation Knowledge, Ámsterdam: John Benjamins, 235-246.

Rundle, Christopher, y Vicente Rafael (20I6): «History and Translation. The Event of Lan-

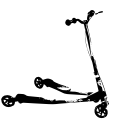

109 
guage», en Yves Gambier y Luc van Doorslaer (eds.), Border Crossings. Translation Studies and Other Disciplines, Ámsterdam/Filadelfia: John Benjamins, 23-48.

110
Thompson, Edward Palmer (I966): «History from Below», The Times Literary Supplement, 7 de abril, 279-280.

Thompson, Paul (1978): The Voice of the Past. Oral History, Oxford: Oxford University Press.

Trouillot, Michel-Rolph (1995): Silencing the Past. Power and the Production of History, Boston: Beacon Press.

Vargas Llos a, Mario (2015): «Reflexiones de un escritor» (Discurso de investidura como Doctor Honoris Causa por la Universidad de Salamanca), Universidad de Salamanca, Sala de Prensa, <http:// www.rae.es/sites/default/files/Discurso_Mario_ Vargas_Llosa_investidura_honoris_causa_Salamanca_septiembre_20I5.pdf $>$ [consulta: 2-V-20I8].
Vidal Claramonte, Ma Carmen África (2018): La traducción y la(s) historia(s). Nuevas vias para la investigación, Granada: Comares.

White, Hayden (1975): Metahistory, Baltimore/ Londres: Johns Hopkins University Press.

White, Hayden (1978a): Tropics of Discourse. Essays in Cultural Criticism, Baltimore/ Londres: Johns Hopkins University Press.

White, Hayden (I978b): «The Historical Text as Literary Artifact», en Robert H. Canary y Henry Kozicki (eds.), The Writing of History, Wisconsin: The University of Wisconsin Press, 4I-62.

White, Hayden (1987): The Content of the Form. Narrative Discourse and Historical Representation, Baltimore/Londres: The Johns Hopkins University Press.

ZARo, Juan Jesús (20I4): «Los hijos de Sánchez», El Trujamán, 3 de febrero <http://cvc.cervantes.es/ trujaman/> [consulta: $2-\mathrm{V}-2 \mathrm{OI} 8]$. 